\title{
Effects of Muscle Energy Technique in Increasing Range of Motion and Strength of Glenohumeral Internal Rotator, in Athletes with Glenohumeral Internal Rotation Deficit
}

\author{
Sonakshi Sehgal, Siddhartha Sen, Amit Dhawan \\ Department of Physiotherapy, Sardar Bhagwan Singh (PG) Institute of Biomedical Sciences \& Research, Dehradun, India \\ Email address: \\ Siddhartha.pt@gmail.com (S. Sen)
}

\section{To cite this article:}

Sonakshi Sehgal, Siddhartha Sen, Amit Dhawan. Effects of Muscle Energy Technique in Increasing Range of Motion and Strength of Glenohumeral Internal Rotator, in Athletes with Glenohumeral Internal Rotation Deficit. American Journal of Sports Science. Vol. 4, No. 2, 2016, pp. 43-48. doi: 10.11648/j.ajss.20160402.14

Received: February 3, 2016; Accepted: February 19, 2016; Published: March 28, 2016

\begin{abstract}
Study design: Randomized control trial. Objective: To measure the effect muscle energy technique(MET) for the glenohumeral joint external rotators to improve the range of motion and strength of internal rotators in athletes with glenohumeral internal rotation deficit (GIRD). Background: Glenohumeral internal rotation deficit is one of the leading causes of impairments in overhead throwing athletes. Anatomical GIRD is though considered to be normal in overhead athletes but it may further lead to osseous and capsuloligamentous changes in the glenohumeral joint if not treated. The muscle energy technique protocol aimed at improving the range of motion and strength of the internal rotators of the glenohumeral joint. It has been hypothesized that MET will increase the glenohumeral internal rotation ROM and strength in athletes with GIRD. Methods: thirty overhead athletes were conveniently assigned in two groups. MET for glenohumeral joint external rotators $(\mathrm{n}=15)$ and stretching for glenohumeral joint external rotators $(\mathrm{n}=15)$. We measured the range of motion of glenohumeral joint internal rotation and strength of glenohumeral joint internal rotators, of the dominant shoulder at 0 day, $1^{\text {st }}$ week and $2^{\text {nd }}$ week. At the end of $2^{\text {nd }}$ week data analysis was done using one way ANOVA and post hoc tukey's test and significant results were found. $(\mathrm{p}<0.05)$. Results: The group treated with MET for the external rotators had a significant increase in internal rotation at the end of $2^{\text {nd }}$ week. The group treated with stretching for external rotators do not showed a significant increase in internal rotation. Conclusion: It is concluded that MET is an effective treatment for increasing the ROM and strength of internal rotation at the glenohumeral joint in asymptomatic overhead athletes. Therefore application of MET for the external rotators may be useful for increasing the ROM as well as strength in overhead athletes.
\end{abstract}

Keywords: Manual Therapy, Athletes, Stretching, Shoulder Joint Internal Rotation

\section{Introduction}

The glenohumeral joint is the most important joint of the shoulder girdle. It is a synovial, ball and socket joint between the humeral head and glenoid fossa of the scapula (William Cornelis 1998). The shoulder joint is stabilized by primary structures, that includes the articular capsule, glenohumeral ligament, and labrum and the secondary structures, that include the rotator cuff, deltoid, and biceps, which also provide dynamic stabilization to the fixed nerve root during throwing motion (Soung Yob R, Wi-Young 2014). From a biomechanical perspective, the glenohumeral joint is typically described as having the following 3 degrees of rotational freedom, that are, plane of motion, elevation, and internal and external rotation (Sean P. McCully et al 2005). Many of the traditional studies of shoulder motion have primarily focused on shoulder elevation, but there has been considerable interest of late, in measuring internal and external rotation along the long axis of the humerus (Reagan KM et al 2002).

Alterations in scapular and humeral kinematics have also been theorized as a result of posterior capsule tightness. Induced posterior capsule tightness in cadavers has resulted in alterations in glenohumeral kinematics, which may place the overhead functioning shoulder at greater risk for injury (Michael $R$. Borich et al 2006). The joint geometry of the glenohumeral joint 
is conductive to excessive mobility but sacrifices osseous stability. (Terry R. Melone, Thomas Mc Poil).

Throwing appears as one of the main gestures which involve this joint being present in many sports such as baseball, handball, tennis, badminton and basketball, with different techniques that depends on each sport. (Valquiria Nunes et al, 2012). Injuries produced by overhead throwing are also the result of an imbalance of two normal force vectors. The internal rotation/flexor muscle groups produce high level of force during acceleration of the arm and so it is necessary that the smaller external rotator muscle group (infraspinatus and teres minor) must counterbalance this by an eccentric contraction (F. H. Savoie, 1993). The joint geometry of the glenohumeral joint is conductive to excessive mobility but sacrifices osseous stability. (Terry $R$. Melone, Thomas Mc Poil). Many researchers have hypothesized that the breakdown in the kinetic chain is one of the reason for shoulder problems. The ground reaction force forms the beginning of the kinetic chain and thus controls the throwing motion. The transfer process in throwing, starts from the ground, to the legs, then the ground, and finally to the ball. (Steven J Karageanes).

Overhead athletes commonly exhibit greater GHJ external rotation $\mathrm{ROM}$ at $90^{\circ}$ of abduction of the dominant arm as compared to the non-dominant arm. However, the total arc of motion (sum of maximum GHJ external rotation and internal rotation $\mathrm{ROM}$ at $90^{\circ}$ of abduction) often does not differ bilaterally, suggesting a corresponding decrease in GHJ internal rotation ROM. This loss of GHJ internal rotation $\mathrm{ROM}$ at $90^{\circ}$ of abduction in the dominant shoulder is referred to as glenohumeral internal rotation deficit (GIRD) (Stephanie D. Moore et al 2014). Studies have reported that there is more external rotation (ER) and less internal rotation (IR) at $90^{\circ}$ of abduction in the throwing versus non-throwing shoulders of baseball players (Jacquelyn Downar JM et al, 2005). Increased external rotation ROM coupled with high joint forces can exceed the physiological limits of the shoulder joint, compromising joint stability. When a loss in internal rotation ROM occurs on the dominant limb without an associated increase in external rotation ROM, pathological GIRD is present (Elizabeth E. Hibberd et al, 2014).

GIRD is calculated as the difference in the maximum humeral internal rotation angle between the dominant (throwing) and non-dominant (non-throwing) limbs. A deficit of 10-17 degree of internal rotation is common in the dominant arm of throwing athletes who have not suffered any shoulder injury. GIRD greater than $25^{\circ}$ has been associated with injuries such as superior labral lesions, subacromial impingement, and pathological internal impingement in the throwing shoulder in dictating a need for investigation of preventative and corrective interventions to restore GHJ internal rotation ROM. [4] (Elizabeth E. Hibberd et al, 2014).

Adaptive changes to bone and soft tissue, occur as a result of the repetitive throwing motion that contribute to the presence of GIRD in the overhead athlete (Stephanie D. Moore et al, 2014). There are many hypothesis on the etiology of the deficit of the medial rotation, one of them states that, it is a result of a contracture and thickening of the postero-inferior portion of the glenohumeral capsule, which occurs due to the repetitive micro trauma during the phases of late cocking and follow through of the throwing movement (Valquiria Nunes et al, 2012).

GIRD causes humeral retroversion, hyperplasia of the joint capsule, and muscular stiffness; and it also induces imbalance in the soft tissue (Jinyoung Lee, Li-Na Kim, Hongsun Song, Sunghwan Kim, 2015). Due to the influence of tightness of the posterior glenohumeral soft tissues on glenohumeral and shoulder kinematics stretching of the posterior glenohumeral tissues to restore glenohumeral IR ROM is a common aspect of shoulder rehabilitation (Jason B. Lunden, Mike Muffenbier, M. Russell Giveans, Cort J. Cieminski, 2010)

Muscle energy techniques are a class of soft tissue osteopathic (originally) manipulation methods that incorporate precisely directed and controlled, patient initiated, isometric and/or isotonic contractions, designed to improve musculoskeletal function and reduce pain(Lean Chaitow). MET may be used to decrease pain, stretch tight muscles and fascia, reduce muscle tonus, improve local circulation, strengthen weak musculature and mobilise joint restrictions (Richa Mahajan, Chitra Kataria, Kshitija Bansal, 2012). This leads to improved postural alignment and the restoration of proper joint biomechanics and functional movement. (B. Chakradhar Reddy, Santosh Metgud 2014). The purpose of the study is to see the effect of MET in increasing the ROM and strength of internal rotators of the glenohumeral joint.

\section{Methodology}

\subsection{Participants}

Thirty subjects between the ages of 16-30 years were included, according to inclusion criteria i.e. subjects who were overthrowing athletes with loss of internal rotation ROM of less than 18 degree to 20 degree in the dominant shoulder as compared to the non-dominant one. The entire subjects were randomly assigned into two groups. The groupA receives MET treatment with hot pack three times a week for 2 weeks while group-B receives stretching with hot pack three times a week for 2 weeks. Glenehumeral internal rotation $\&$ isometric strength of internal rotator muscle was measured as outcome measure before the starting of treatment and after $1^{\text {st }} \& 2^{\text {nd }}$ week.

\subsection{Procedures}

Measurement of Glenohumeral Internal Rotation: The subjects were positioned supine on a treatment table with a hip and knee flexed to 90 degree. The shoulder to be examined was kept initially at 90 degree of abduction and 90 degree of elbow flexion, with the arm perpendicular to the ground. Stabilization of the scapula was achieved by placing a towel roll below the shoulder. The goniometer was aligned, such that, the axis of the goniometer was the olecranon process of the ulna, the stationary arm was perpendicular to 
the ground and the moving arm was parallel to the longitudinal axis of the ulna pointing towards the styloid process. The shoulder was then, passively internally rotated with one hand, and the ROM measurement was taken at the first point of resistance, using a goniometer. (Fig. 1)

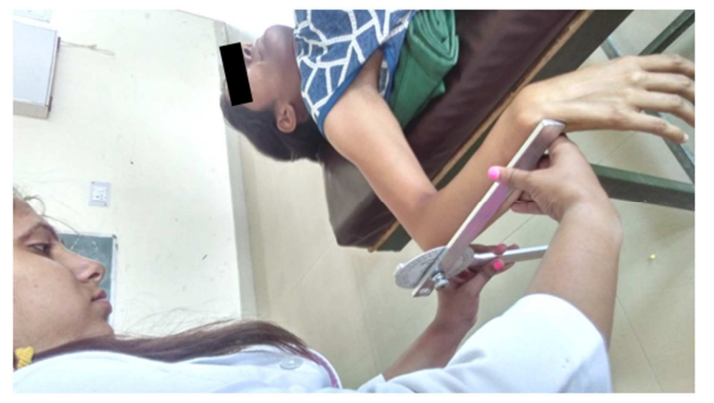

Fig. 1. Showing Measurement of Internal Rotation.

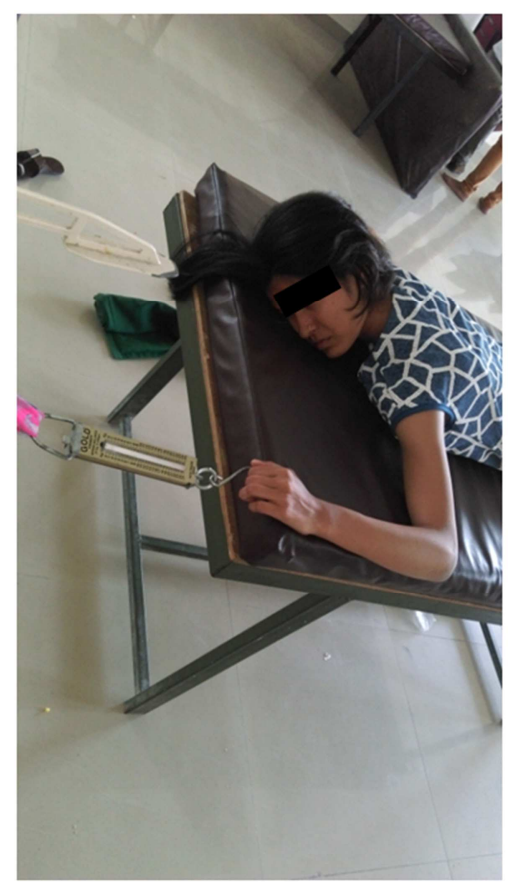

Fig. 2. Showing Measurement of Internal Rotator Strength.

Measurement of Glenohumeral Internal Rotators Strength: The subjects were positioned prone on the treatment table, with the shoulder at 90 degree of abduction. A leather wrist strap was positioned $15 \mathrm{~cm}$ distal to the olecranon process, and was attached to the strain gauge, via, a chain. The subjects were asked to perform maximum voluntary isometric internal rotation contraction in the neutral position. The contraction was sustained for five seconds. The value of the force generated during maximum isometric shoulder internal rotation contraction was recorded in the strain gauge. (D L Falla, S Hess, C Richardson, 2003) (Fig. 2)

Application of Muscle Energy Technique (PIR):- The subject was positioned supine on the treatment table with the shoulder and elbow, at 90 degree of abduction and flexion. The shoulder was stabilized at the acromion process with one hand, and the other hand was used to passively move the arm into internal rotation until the first barrier of motion was reached. The subject was then instructed to perform a 5second isometric contraction of approximately $25 \%$ maximal effort in the direction of external rotation, against an opposing force provided at the distal forearm. Following the contraction, the subject was instructed to internally rotate the arm toward the ground as a 30-second active assisted stretch was applied. The subject was instructed to relax, and a new movement barrier was then engaged. This protocol was performed for a total of 3 repetitions. (Stephanie D. Moore et al 2011). This lengthens the external rotators thus increasing the internal rotation range of motion. (Fig. 3)

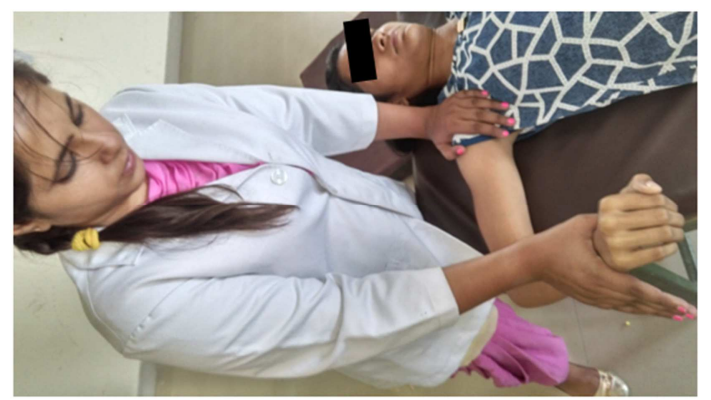

Fig. 3. Showing Application Of MET to the External Rotators.

Application of Stretching:- The subject was positioned supine with the shoulder at 90 degree of abduction and elbow at 90 degree of flexion. The shoulder is stabilized at the acromion with one hand and the arm is passively internally rotated with the other hand. The stretch was maintained for 10 seconds. The protocol was repeated 3 times. This lengthens the external rotators thus increasing the internal rotation range of motion. (Kisner and Colby). (Fig. 4)

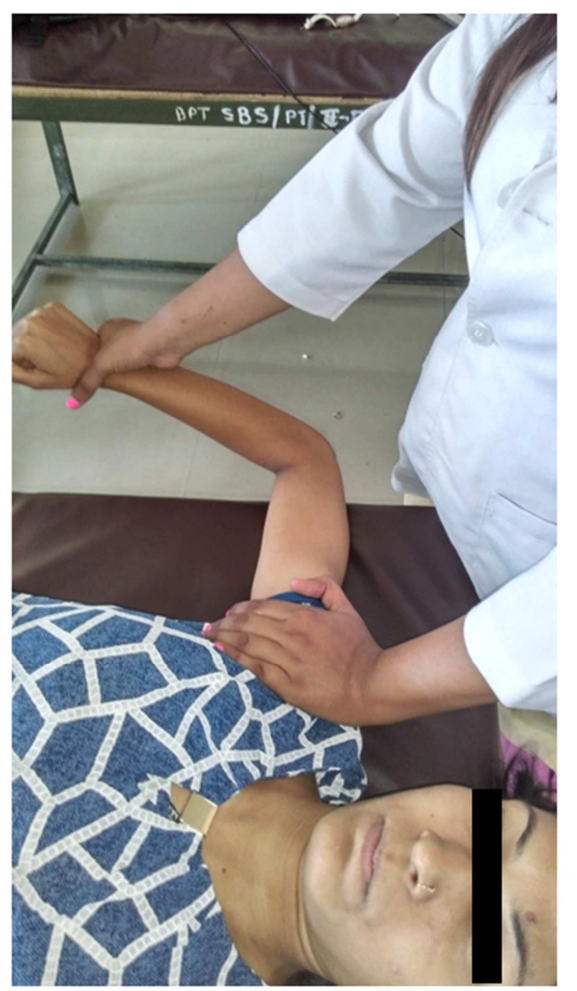

Fig. 4. Showing Application of Stretching To External Rotators. 


\subsection{Data Analysis}

In this study, 30 subjects were taken, two groups were made i.e. A and B and 15 subjects were included in each group. One way ANOVA has been made to compare the ROM and strength in between the groups, in starting day, $1^{\text {st }}$ week and $2^{\text {nd }}$ week. Post hoc analysis was done using turkey's multiple comparison test for ROM and strength in starting day, $1^{\text {st }}$ week and $2^{\text {nd }}$ week. $t$ - test was done for checking the significant level between the groups.

Significant level has been selected as $\mathrm{p}<0.05$.

\section{Results}

t-test analysis showed non- significant result for ROM and strength ( $p=0.3$ and 0.2 )

One way ANOVA was done to compare ROM and strength of glenohumeral internal rotators. The result showed significant differences for ROM and strength in group A $(\mathrm{p}=0.000$ and 0.001$)$ and non-significant result for ROM and strength in group $B(p=0.177$ and 0.543 )

Post hoc turkeys test analysis shown significant result for ROM and strength in group $\mathrm{A}(\mathrm{p}=0.000$ and 0.000$)$ and nonsignificant for ROM and strength in group $\mathrm{B}(\mathrm{p}=0.064$ and $0.273)$.

Table 1. One way ANOVA in MET group.

\begin{tabular}{lll}
\hline & F-value & P-value \\
\hline ROM & 17.605 & 0.00 \\
STRENGTH & 8.536 & 0.001 \\
\hline
\end{tabular}

Table 2. One way ANOVA in Stretching group.

\begin{tabular}{lll}
\hline & F-value & P-value \\
\hline ROM & 1.808 & 0.177 \\
STRENGTH & 0.620 & 0.543 \\
\hline
\end{tabular}

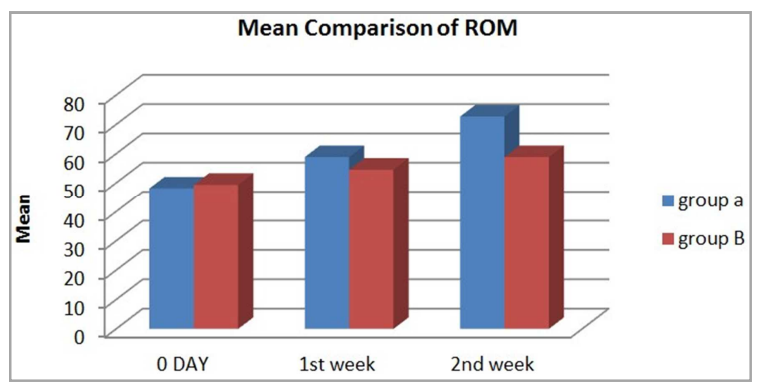

Fig. 5. Mean comparison of ROM between group A and group B at o day, $I^{\text {st }}$ week and $2^{\text {nd }}$ week.

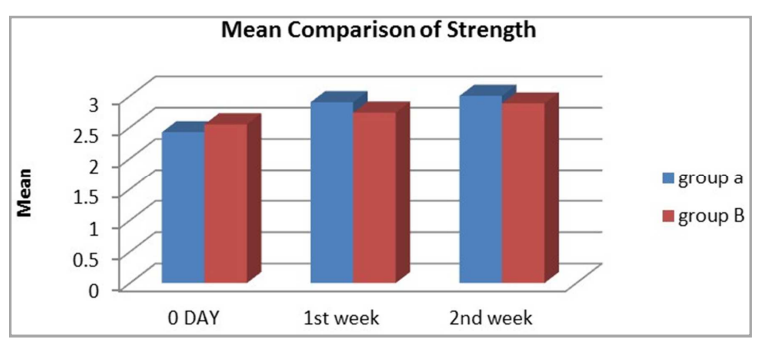

Fig. 6. Mean comparison of Strength between group A and group B at o day, $1^{\text {st }}$ week and $2^{\text {nd }}$ week.

\section{Discussion}

The recent study, demonstrated a significant relationship between GIRD and pain, isokinetic strength, and the quality of life, among domestic high school male baseball players. (Jinyoung Lee, Li-Na Kim, Hongsun Song et al, 2015). The decrease in glenohumeral internal rotation in overhead athletes in the dominant shoulder is also noted in past researches (Stephanie D. Moore Et Al 2014). The internal rotation ROM and strength in the throwing shoulder is limited due to osseous adaptation, posterior muscle tightness and posterior inferior capsule tightness.

In our study we observed that the resistance felt at the end range of motion had a rubbery end feel suggesting that the participant may had deficit in the internal rotation due to soft tissue tightness. The participants taken in the study had a pain free shoulder and were having anatomical GIRD.

There is also a significant change in kinematics, during the throwing motion. In a kinetic chain the energy and momentum are transferred through adjoining body segments via coordinated motion resulting in maximum transfer to the terminal segment and thus a throwing motion a kinetic chain is consists of the legs and hips trunk, shoulder, upper arm, forearm and hand, with the ball serving as the terminal segment, use of all the components of the kinetic chain allows the throwers to attain maximum performance without causing undue overload to the shoulder. A failure or fatigue of one or more components of the kinetic chain places increase demand on the remaining chain and causes lack of internal rotation strength.

In our present study we found that the change in the kinematic chain could be due to the fatigue in the soft tissue structures in the glenohumeral joint. The fatigue in the muscles and ligaments would have placed stress on the shoulder due to which there was a loss in the internal rotation of the dominant shoulder, but the participant do not have shoulder pain which clearly depicts that osseous changes did not took place in the glenohumeral joint.

Many researchers have explained the different phases of throwing, in the glenohumeral joint. Every phase have its different biomechanics and force production by the muscle which act during the motion of the shoulder. During the cocking phase of pitching and throwing, the high level of loading on the shoulder passive restraints causes gradual stretching of the capsular collagen leading to an increase in external rotation ROM (Elizabeth E. Hibberd et al, 2014). During the acceleration phase of throwing, the supraspinatus, infraspinatus and teres minor work eccentrically to provide stability to the humeral head and thus prevent excessive anterior translation of the humeral head (Juneja H, Verma, S. K., Khanna, 2011). During the throwing motion, the centre of rotation is shifted postero superiorly on the glenoid and this alters the interplay of torsional forces on the superior labrum and related biceps tendon and anchor. This allows for further external rotation by increasing the clearance for the greater tuberosity and also prevents direct internal impingement of the posterosuperior labrum/rotator cuff. Furthermore if this 
biomechanics is altered, anterior capsular laxity and loss of glenohumeral internal rotation in abduction occur, resulting in a thrower's "dead-arm" (Hugue Ouellette et al, 2007). In one the study Burkhart et al believed that athletes with glenohumeral internal rotation deficit exhibit a posterosuperior shift increasing contact among the humeral head, labrum, and rotator cuff in the late cocking phase of the throwing shoulder (Robert C Manske RC, et al, 2010).

On the contrary, in our present study we found that there was a change in internal and external rotation, bilaterally but the total arc of motion was not affected which clearly suggest that mild changes which were present in the soft tissues of the throwing athlete were due to the alteration in joint biomechanics and imbalance in the muscles.

In our present study, the measurement of the ROM of internal rotation was done in the supine lying position, as this position is more reliable and comfortable for the subject. The supine lying position also provides scapular stabilization and thus decreases error during examination.

In the past research, it was demonstrated that a single application of MET for the GHJ horizontal abductors results in greater post treatment GHJ horizontal adduction and internal rotation ROM immediately following treatment. Alternatively a single application of MET applied to the GHJ external rotators did not lead to a significant increase in horizontal adduction or internal rotation (Stephanie D. Moore Et Al 2014).

Roberts indicated the effects of MET as decreased pain, increased range of motion, decreased muscle tension and spasm, and increased strength. Another study by, Greenman (1989) depicts that Muscle Energy Technique helps to regain the mobility of the hypomobile joints by restoring normal length tension relationships which are shortened and by strengthening the weakened muscles and reduce edema by pumping action for lymphatic system(B. Chakradhar Reddy, Santosh Metgud, 2014)

One of the experimental study also concluded that MET produced a change in ROM was possibly due to an increased tolerance to stretch, as there was no evidence of Viscoelastic change (B. Chakradhar Reddy, Santosh Metgud, 2014).

According to Lean Chaitow the physiological mechanisms behind the changes in muscle extensibility produced by MET are reflex relaxation, viscoelastic or muscle property change, and changes to stretch tolerance - a change to tolerance to stretching is most supported by the scientific literature (lean Chaitow). These mechanisms bring about a change in muscle physiology and hence lead to increased ROM at the joint.

In the present study, we demonstrated that an application of MET on GHJ external rotators increases the ROM and strength of internal rotators. This increase in ROM and strength of glenohumeral internal rotators was due to change in the muscle extensibility followed by relaxation of the muscle. The isometric contraction of the muscle is thought to increase the strength of the muscles.

On the other hand, the group which was treated with stretching of the external rotators did not show a significant increase in the GHJ internal rotators ROM and strength.
Stretching is thought to increase the flexibility at the joint, as proved in many of the past researches.

In the past study Bukhart, et al, described a sleeper stretch to be effective in increasing the range of motion of the glenohumeral joint in throwing athletes. A cross body stretch is also found to be effective in increasing posterior shoulder soft tissue flexibility (Kevin E. Wilk, Todd R. Hooks, Leonard C. Macrina, 2013).

One of the study demonstrated that stretching protocol has the ability to the increase the shoulder internal rotation and total arc of motion in the throwing shoulder. (Roy Aldridge, J. Stephen Guffey, Malcolm T. Whitehead, Penny Head, 2012).

Though may studies support that stretching is effective in improving the ROM at the joint, but in the present study it is non-significant when compared to the MET. This may be, because the protocol was given 3 days in week, which may not be able to bring effective outcomes.

As a result the present study proved that MET is effective in increasing the ROM and strength of internal rotation of the glenohumeral joint. Flexibility is considered to be a valuable component of athletic performance, so the subjects involved in overhead throwing sports can be treated with MET to improve the flexibility.

\section{Conclusion}

MET was used to treat limited glenohumeral internal rotation in athletes with GIRD. The study concluded that the treatment showed remarked improvement in the range of motion and strength of internal rotators in athletes with GIRD. Therefore the technique can be used in acute cases of posterior shoulder tightness to prevent the occurrence of other shoulder injuries.

\section{References}

[1] B. Chakradhar Reddy, Santosh Metgud, A randomized controlled trial to compare the effect of muscle energy technique with conventional therapy in stage ii adhesive capsulitis, 2014 Int J Physiother Res; 2(3): 549-54.

[2] Charles I. Dillman, Biomechanics of Pitching with Emphasis upon Shoulder Kinematics, 1993 J Orthop Sports Phys Ther; 18: 402-408.

[3] D L Falla, S Hess, C Richardson, Evaluation of shoulder internal rotator muscle strength in baseball players with physical signs of glenohumeral joint instability, 2003, Br J Sports Med; 37: 430 - 432.

[4] Elizabeth E. Hibberd, Casey E. Shutt, Sakiko Oyama, Physical contributors to glenohumeral internal rotation deficit in high school baseball players, 2014, Journal of Sport and Health Science 1-8.

[5] F. H. Savoie, Arthroscopic Examination of the Throwing Shoulder, 1993 JOSPT; 18: 409-41.

[6] Hugue Ouellette et al, Spectrum of shoulder injuries in the baseball pitcher, 2007 Skeletal Radiol; 37: 491 - 498. 
[7] Jacquelyn M. Downar; Eric L. Sauers, Clinical measures of shoulder mobility in the professional baseball player, 2005 Journal of Athletic Training; 40(1): $23-29$.

[8] Jason B. Lunden, Mike Muffenbier, M. Russell Giveans, Cort J. Cieminski, Reliability of Shoulder Internal Rotation Passive Range of Motion Measurements in the Supine Versus Sidelying Position, 2010, J Orthop Sports Physical Therapy; 40: 589-594.

[9] Jinyoung Lee, Li-Na Kim, Hongsun Song, Sunghwan Kim, The Effect of Glenohumeral Internal Rotation Deficit on the Isokinetic Strength, Pain, and Quality of Life in Male High School Baseball Players, 2015, Ann Rehabil Med; 39(2): 183190.

[10] Juneja H, Verma, S. K., Khanna,, Isometric Peak Force of Shoulder Rotators in Cricketers with and without History of Shoulder Pain, 2011 Journal of Exercise Science and Physiotherapy; 7 (1): 42-49.

[11] Kevin E. Wilk, Todd R. Hooks, Leonard C. Macrina, 2013, J Orthop Sports Phys Ther; 43: 891-894.

[12] Kisner And Colby, Therapeutic exercise foundation and technique, 5 th edition.

[13] Leandro Anonietti, Natalia Luna, Gabreil Nogueira et al, Reliability Index of inter- and intra-rater of manual goniometry and computerized biophotogrammetry to assess the range of motion of internal and external shoulder rotation, 2014 Medical express; 1(2): 95-99.

[14] Lean Chaitow, Musc e Energy Techniques, third edition, London UK; Churchill Livingstone page no. 1.

[15] Mark G Grossman MG, A Cadaveric Model of the Throwing Shoulder: A Possible Etiology of Superior Labrum Anterior-toPosterior Lesions, 2005 J Bone Joint Surg Am; 87 (4): 824-831.

[16] Michael R. Borich, Scapular angular positioning at end range internal rotation in cases of glenohumeral internal rotation deficit2006 J Orthop Sports Phys Ther; 36(12): 926-934.

[17] Reagan KM, humeral retroversion and its relationship to glenohumeral rotation in the shoulder of college baseball player, 2002, The American Orthopaedic Society for Sports Medicine.
[18] Richa Mahajan, Chitra Kataria, Kshitija Bansal, Comparative effectiveness of Muscle Energy Technique and static stretching for treatment of subacute mechanical neck pain, 2012, International Journal of Health and Rehabilitation Sciences; 1 (1): 16-23.

[19] Robert C. Manske, Matt Meschke, Andrew Porter, A Randomized Controlled Single-Blinded Comparison of Stretching Versus Stretching and Joint Mobilization for Posterior Shoulder Tightness Measured by Internal Rotation Motion Loss, 2010 Sports Health, vol 2, no. 2.

[20] Roy Aldridge, J. Stephen Guffey, Malcolm T. Whitehead, Penny Head, 2012, The International Journal of Sports Physical Therapy; 7(4): 365.

[21] Russel F. Warren, Edward V Craig, David W. Altchek, The unstable shoulder, Lippincot.

[22] Sean P. McCully, Internal and external rotation of the shoulder: effects of plane, end-range determination, and scapular motion, 2005, J Shoulder Elbow Surg pg: 602-610.

[23] Soung Yob R, Wi-Young, Analysis of Range of Motion and Isokinetic Strength of Internal and External Rotation According to Humeral Retroversion of the Dominant Shoulder in Youth Baseball Players, Iranian J Publ Health, 2014; 43: 178-184.

[24] Stephanie D. Moore, Kevin G. Laudner, Todd A. Mcloda,, The Immediate Effects of Muscle Energy Technique on Posterior Shoulder Tightness, 2014 Journal of orthopaedic \& sports physical therapy; 41: 400-407.

[25] Steven J Karageanes, Principles of Manual Sports Medicine, Lippincott William \& Wilkin.

[26] Terry R. Melone, Thomas Mc Poil, Orthopaedics And Sports Physical Therapy, Third Edition.

[27] Valquiria Nunes, Assessment of deficit in medial rotation and posterior shortening of the shoulder in professional basketball player, 2012 Rev Bras Med Esporte - Vol. 18, No 3.

[28] William Cornelis, Biomechanical Relationships within the Shoulder Joint. USA Gymnastics Online: Technique, May 1998, Vol. 18, No. 5. 